Original Research Paper

\title{
Medication Errors: Preventing Untimely Deaths
}

\author{
${ }^{1,3}$ Cheryl Ann Alexander and ${ }^{2}$ Lidong Wang \\ ${ }^{I}$ Department of Nursing, University of Phoenix, USA \\ ${ }^{2}$ Department of Engineering Technology, Mississippi Valley State University, USA \\ ${ }^{3}$ Technology and Healthcare Solutions, Inc., USA
}

\author{
Article history \\ Received: 24-09-2014 \\ Revised: 03-10-2014 \\ Accepted: 16-12-2015 \\ Corresponding Author: \\ Lidong Wang, \\ Department of Engineering \\ Technology, Mississippi Valley \\ State University, \\ USA \\ Email: 1wang22@students.tntech.edu
}

\begin{abstract}
Medication adverse events play a huge role in hospital deaths and morbidity in the US. This plays a large role in the public health of the US at large. Health education is a primary target for those taking medications. A quality assurance or improvement program that is appropriate to capture drug events and measure quality data is essential to prevent these untimely and mostly preventable deaths. This study examines medication errors, the cause and effect and what leaders can do to prevent untimely patient deaths.
\end{abstract}

Keywords: Medication Errors, Information Technology, Quality Improvement, Medication Adverse Events, Health Education, Logistics and Quality Management, Public Health, Health Management

\section{Introduction}

Medications are a substantial element of care for patients over 65 . Elderly patients are prescribed more than $30 \%$ of all prescription drugs. Adverse drug events or reactions to medicines are implicated in 5\% to $17 \%$ of inpatient admissions, but even more medication difficulties could be prevented by monitoring drug therapy and identifying patients at risk (NQMC, 2011). Medication use in the patient over age 65 is a significant topic for research and directed Quality Improvement (QI) in clinical practice. There are more than 2 million severe adverse drug reactions annually and about 100,000 deaths due to medication problems. Noncompliance, non-adherence, the difficulty of communication between patients and providers and the burden of taking multiple medications can result in drug interactions, adverse drug events, drug overuse and drug underuse. Adverse drug events are a leading cause of morbidity and mortality. As of January 2009, the Institute of Medicine (IOM) reported that annually between 44,000 and 98,000 deaths result from medical errors; while an estimated 7,000 deaths result from adverse drug reactions. If medication reconciliations were used however, adverse drug events can be targeted. In one such study, the use of medication reconciliation forms led to a drop in the percentage of patients affected by adverse drug events from $36.9 \%$ to $9.3 \%$ (NQMC, 2011).

Nurses play a key role in administering medications for inpatients. Some reports identified errors by nurses as
1 in $5(19 \%)$ medication administrations. A study of workflow related to medication management by nurses reported that $27 \%$ of nurses' time was spent on medication-related activities. This observational study further found major aspects of medication-related activities as follows: Obtaining and verifying medications $7.4 \%$, medication delivery $6.7 \%$, information retrieval $3.9 \%$, management of physician orders $3.9 \%$ and documentation of medication administration $2.8 \%$. Medication administration is a complicated procedure frequently performed by nurses and the opening for error increases significantly with the number of medications to be administered and patient acuity (Johnson and Young, 2011).

In an environment of limited resources and high demands on improving healthcare quality, many providers and organizations alike worry that transparency of processes and outcomes may lead to retribution, decline in referrals (whether self-referrals or other provider referrals) and presentation of wrong data. To address the worry of retribution, organizations must encourage transparency and open reporting so that individuals can learn from their mistakes and provide appropriate assurances that outcomes will not be used for disciplinary action. Data for accountability and pay performance can be seriously counted and expectations set (Meredith, 2011). However, the expectations must be appropriate and fair. Presentation of correct, risk-adjusted data with comparison or benchmarking with like organizations is necessary to earn staff buy-in and support for 
transparency. Dashboards, scorecards, or report cards often used for financial or operational measures are also useful in quality measures. While real-time may not be as significant for outcome measures as for operational ones, timeliness is a must. Providing information to the staff at the point of care helps detect problematic areas and gains staff buy-in, improvement and sustainment (Meredith, 2011). With the right performance improvement program, the agency will have a head start on improving its processes.

As the baby-boomers age, the elderly are deciding to stay in their own homes rather than seek care in a residential home or nursing home. The demand for nurses and medication management in the home based environment is most dire. On average, most residents are taking between five and seven medications. Medication management therefore, in a nursing home, takes up approximately $47 \%$ of staff's time. Proper dispensation of medication is necessary to ensure safety for residents and nurses. Quality indicators need to be examined carefully to determine what tool is used to measure successful medication runs. For home-based patients, home health nurses need a reliable measurement tool for quality assurance as well. Although they are not directly handling medications three or four times daily, these home health nurses are teaching about medications and side effects (Wright, 2014). A discussion on the background and problem, review of quality indicator process and an explanation of the quality indicator chosen for a case study on medication administration to prevent medication errors will be presented in this study.

\section{Logistics and Quality Management}

Health care is still quality-driven and is in a crisis even after introducing quality management. The last two National Healthcare Quality Reports have indicated that quality improvements have been slow and subject to noteworthy unpredictability across regions and settings. Further, a recent analysis by the CMS predicted that $13.5 \%$ of Medicare beneficiaries experienced an adverse event during a hospital stay (Glasgow, 2011). This lack of improvement is concerning, especially in any hospitals where Quality Improvement (QI) is a universal activity. This raises the question of whether current QI methods are not sufficient for effecting widespread change. Plan-Do-Study-Act (PDSA) is the leading cycle that forms the basis of most QI activities. The PDSA cycle is akin to the scientific approach because it presents an overlying background for approaching a problem, but does not plainly exclude the best methods for solving a problem. Lean and Six Sigma put PDSA within a specific context that should present a directed focus for optimizing the QI process (Glasgow, 2011).

\section{Public Health Management}

A frequent solution that improves quality in health care is to round up a group of people, tell them what they are not doing right, train them how they could do better and return them to work. Sometimes, if appropriately directed, this educational method may result in initial improvements, but in the hectic world of health care, it is too easy to unconsciously return to old ways. The failure of simple education is exemplified to use the education of nurses to reduce medication mistakes. While the intervention made initial improvement, within two years of the intervention not only were all improvements lost, but the hospital recorded a quarterly rate of medication mistakes higher than any seen in the past four years (Glasgow, 2011).

Several adverse drug events can occur with topical analgesic patches. A study was conducted to determine quality indicators in the environment that would have the potential to identify probable risk factors for patients using topical patches such as fentanyl and buprenorphine. All incidents concerning drug reports were consolidated from 2004-2011 to a regional reporting system. Pharmacy records were used to determine adverse events. It seemed to be an effective system for keeping track of adverse events for the facility. The three most common errors were wrong administration time, wrong dose and omission of dose (Lövborg et al., 2014).

\section{Health Education}

Change is very lasting when the individuals who provide frontline care are involved and really excited about the QI they helped develop. While it is very significant that the individuals who make the strategic plan for an organization make it clear that they value and support QI, the health care system does not apply itself to the necessary carrots and sticks for management to easily affect change. Instead, it must be the frontline leaders who identify a problem in quality, explain the need for a change and inspire individuals around them to meet and overcome the challenge. Furthermore, it is the individuals who understand how a process really occurs and can best detect the waste or potential sources of mistake. Only when there is real energy at the front lines for facilitating a change is it possible to obtain long-term quality (Glasgow, 2011).

In one Quebec hospital, there were 37 adverse events, however, at that time 10 of the events were fatal. In nine out of 10 emergency department visits, seven were related to medication events and at least $70 \%$ were preventable. Nurses can be proactive by educating patients prior to discharge, at medication administration and providing educational sessions during inpatient stays, particularly for insulin. To gain a better perspective, E-safe files were created for adverse events, flagging them as they occurred (Au et al., 2014). Figure 1 (Arinal et al., 2014) indicates a nurse placing medications in a lock box within the patient's room in a hospital. 


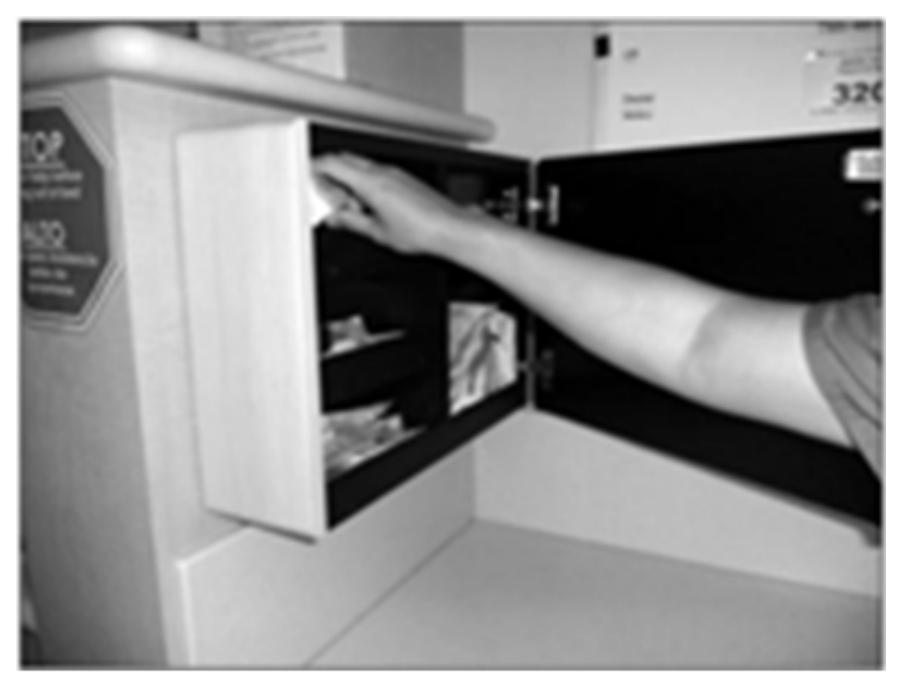

Fig. 1. Medication cabinet and stocked supplies

\section{Errors and Economics}

For administrators and nurse leaders, cutting medication errors is essential to safe patient care and compliance with the Joint Commission (JCAHO), CMS standards and third party payers. There is no reimbursement to care for the patient who is a victim of a medication error (JC, 2012) and the CEO must closely watch the financial viability of the facility as well as the reputation. Beyond that, the patient is the most important and subjecting patients to poor medical care is the most important economic factor to avoid. The Joint Commission has standards of care set in National Patient Safety Goals for medication management. First the nurse must identify the patient by two identifiers such as name and birthdate. All medications in basins, syringes and cups must be labeled. Extra precautions are used with patients receiving blood thinners. The nurse is to record and reconcile correctly a patient's medications prior to admission and at discharge (JC, 2012). These environmental musts are necessary to ensure quality care for the inpatient.

\section{Market in Error Advocacy}

The hospital with the least amount of published errors is going to be the one with highest population of patients. If a hospital has a high inpatient medication error rate, the quality of care is not very high and patients will most likely request to be admitted elsewhere. A marketable image is more than valuable in health care.

The World Health Organization (WHO), in conjunction with many agencies from around the world, is promoting pharmacovigilence. Pharmacoviligence (PV) involves quickly targeting medication errors and training staff and appropriate personnel how to avoid the errors in the future. It also involves promoting a system of early identification of situations that could lead to medication errors such as name alerts, critical care units where many errors happen and insulin. To market this approach, the WHO PV marketing should be geared towards behavioral changes that will promote adverse drug reaction (ADR) reporting (WHO, 2011). Marketing can be enhanced using the media and other public initiatives. The impact of PV marketing efforts should be measured by analyzing prescription data before and after, analyzing media coverage and assessing behavioral changes (WHO, 2011).

\section{Cultural Factors which Influence Error Rate}

Health care will never be made substantially safer by focusing after the fact on individual providers. Additionally, naming, blaming and shaming individuals for doing things we are all capable of, never makes a system safer-and may have the opposite effect. Health care organizations that try to restore public confidence by punishing individuals suffer from the "vulnerable system syndrome." (Flemons and Avies, 2011). The syndrome is characterized by three elements: Blaming frontline individuals, denying the potential for catastrophes and mistakenly pursuing the "wrong type of excellence" with a focus on production and finance performance targets. That does not mean that evaluating post-event performance is not significant. However, the most important cultural aspect of this is the "culture of safety". Through continual evaluation and performance improvement, a "culture of safety" can be cultivated by preventing errors before they occur. An organization must have the ability to have a reporting system that includes close calls and actual events. While having a just culture means reprimanding those who ignore organizational standards or intentionally harm patients, it also means having recourse to remediate those who make mistakes. People within the organization should be 
commended for providing information related to medication errors, not judged harshly. A just culture also means having the means that organizations should have the ability to draw conclusions about what is needed in terms of reform. One example is that power may shift to the top level executive during a time of Crisis (CEO). Organizations that have evolved this way have developed a flexible culture (Flemons and Davies, 2011).

\section{Politics that Influence Errors}

The use of Electronic Health Records (EHRs) has received a lot of attention during the last decade. In the US, the Obama administration is earmarking \$50 billion dollars for health IT to capitalize on the documented efficiencies and safety improvements EHR implementations can bring. Safety improvements, better healthcare quality and reduced costs are several of the advantages of implementing EHRs (Cannoy and Carter, 2011). The focus of the Cannoy and Carter study was the human network of stakeholders who must cooperate to decide the policies, procedures, laws and technological features for how EHR information will be exchanged electronically. EHRs increase the safety of medication administration by putting in place safety checks that people who administer medications cannot avoid. The Cannoy and Carter research focuses on the information politics related to EHR implementations in complicated network environments. Politics involves how individuals negotiate decisions and objectives through relationships of control and influence. It has been shown that politics plays a key role in health information networks (Cannoy and Carter, 2011).

\section{Review of Quality Indicator Process}

Performance reporting is important in contemporary organizations. Iselin et al. (2011) suggest that the choice of performance indicators is one of the most critical challenges facing organizations. Performance reporting is significant in motivating employees to improve performance and is a necessary element of an organization's control system. Iselin et al. (2011) argue that present performance reporting does not cover information about future performance drivers, such as customer satisfaction, efficiency of the internal process and employee morale and capabilities. Performance reporting has traditionally been in financial terms. However, there has been more and more criticism of financial reporting. The main criticisms are that present financial reporting is historic and is not a reasonable predictor of future financial performance. It is a reference to examples of past main corporate collapses in which present financial information did not indicate impending financial disaster. There are a number of reasons managers might adopt a traditional performance improvement program. Evidence-based practice indicates that these programs are reliable and can indicate problems areas where they exist (Iselin et al., 2011). Managers must be vigilant in applying evidence-based practice in that they choose a workable and theoretically sound model for use in their facility.

McMillian (2011) argues that performance improvement for managers today has improved over the years to include more pertinent data and better collection processes. Having data collected that is pertinent to the organization's daily function is important because that is how managers will see problems develop and be able to intervene before the problems become too advanced. Retrospective factors such as reviewing the case after the fact and finding evidence that targeted behaviors were in error can be useful in improving care. The same can be same for prospective and concurrent reviews. With prospective reviews, reviewing the case before something happens can prevent a costly error and managers can rest easy knowing a crisis has been averted (McMillian, 2011). For medication errors, it would be best to have a prospective review if possible to prevent errors from occurring, however, most likely the majority of medication errors fall under retrospective reviews. Managers need to take action review documentation, look at medication administration techniques and other factors involved in medicating patients. It is only then will prospective reviews be effective.

\section{Medication Errors and Information Technology}

In 2001, the Institute for Medicine announced that Information Technology (IT) is going to be critical to quality improvement and data collection with positive outcomes in the coming years. This prediction foretells the electronic medical record and data gathering within the technology. Computerized order entry is proposed to make medication administration safer for patients; for example, problems with legibility and unclear orders would be eliminated. Early IT focused however, only on medication administration using barcodes and ultimately Radio Frequency Identification (RFID) technology (Cheung et al., 2014).

In current health care, infusion pumps, ventilators and other interfacing data systems will help manage medication safety. Quality indicators for patients, residents and home patients is to mine the data gathered from medication administration logs, order entry $\log s$ and as needed medication logs. Getting quality data for QI means policing the IT system for errors committed by the human users (Cheung et al., 2014). Figure 2 shows the process of medication error prevention (Carayon et al., 2014). 


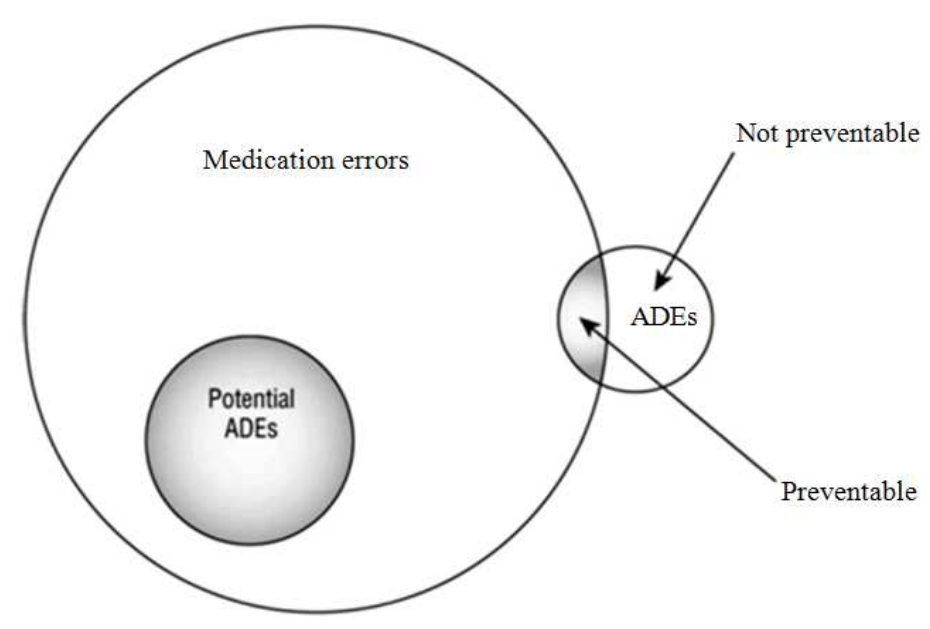

Fig. 2. The process of medication error prevention (ADEs: adverse drug events)

Clinical indicators evaluate particular health structures, processes and outcomes. They can be rate- or mean-based, presenting a quantitative basis for improvement in quality, or sentinel, detecting incidents of care that trigger further investigation. They can evaluate aspects of the structure, process, or outcome of health care. Additionally, indicators can be generic measures that are related to most patients or diseasespecific, indicating the care quality for patients with specific diagnoses (Mainz, 2003). Evaluating the care quality has become more and more significant to providers, regulators and purchasers of care. Recently, providers were interested in evidence-based medicine and purchasers began to focus on the cost-effectiveness of health care in generating health outcomes. Indicators for performance and outcome assessment allow the quality of care and services to be evaluated. This evaluation can be performed by creating quality indicators that measure the performance that occurs for particular patients or relevant health outcomes and then assessing whether patients' care is consistent with the indicators according to evidence-based standards of care. Indicator measurement and monitoring can have many purposes. It has been made possible to: Document the quality of care; perform comparisons (benchmarking) over time between places (hospitals); perform judgments and set priorities (selecting a hospital or surgery, or organizing medical care); facilitate accountability, regulation and accreditation; make quality improvement; and respect patient choice of providers (Mainz, 2003). Indicators can make professionals and organizations monitor and assess what happens to patients resulting from how well professionals and organizational systems function to meet the patients' needs. But indicators are not a direct quality measure because quality is multidimensional and many different measures are required for understanding quality well.

The indicator most often used to measure for medication errors is adverse drug events. Adverse drug events occur after a patient takes a wrong medication and has an adverse reaction to it. A study by Hamrick et al. (2009) found that $78 \%$ of clinicians were not responding to questions related to patient drugs upon administration. At least $68 \%$ were not informing patients of new medications and $51 \%$ were not informing patients of medication changes at all (Hamrick et al., 2009). These alarming rates identify a problem where clinicians are simply not taking the time to answer questions and involve the patient in their own care. A simple thing like this could cut out many drug errors. However, the Hamrick et al. (2009) study found that the one indicator most often looked at for medication errors was the adverse drug event forms. Wrongly medicating a patient who does not have an adverse event may or may not go unnoticed. Selfreporting may be on the honor system. More stringent reporting methods should be implemented to allow for better capture of medication error data.

\section{Quality Improvement Plan}

Mississippi Baptist Hospital (MBHS), a for-profit organization which houses approximately 367 patients daily, recognizes the need to be foremost in health care in this current society of health care competition and strict reimbursement. In order to meet these goals, MBHS has issued a new performance improvement program to combat the increase in medication errors. Aligned with the vision of MBHS, the program will help Baptist become the "premier provider of health care in the Jackson area" and meet one of its 10 strategic goals set for 2012 (MBHS, 2011). The MBHS Board of Trustees formed an ad hoc quality measurement review committee that was responsible for determining major health care quality indicators and benchmarks and suggesting implementing plans to evaluate and enhance MBHS quality of care. The ad hoc committee started its 
task before the release of the first report of the Institute of Medicine's (IOM) Committee on Quality of Healthcare in America and finished its response prior to the release of the second IOM report (Ballard, 2003). To guarantee that MBHS quality care programs in the future align with national directions of health care quality, the ad hoc committee applied the 2010 IOM definition of quality care as the degree to which health services for people improve the chance of desired health outcomes and are consistent with today's professional knowledge. The committee combined perspectives of the IOM and the RAND (research and development) organization by creating a health care strategy as one that was considered a must to the extent that it would not be suitable care not to recommend the strategy, that the strategy had a suitable likelihood of providing patients with benefits and that the potential benefits were great (Ballard, 2003). Table 1 (Carayon et al., 2014) illustrates a description of patients and medication errors.

The ad hoc committee report recognized three major elements of an implementation plan to evaluate and enhance quality of care in MBHS primary care centers and hospitals. The first was creating a dedicated MBHS entity to prioritize and commit necessary resources and successfully implement system-wide health care improvement initiatives by assimilating the efforts of administrative, nursing and physician leadership. This resulted in forming the MBHS Best Care Committee, which would develop project concepts, perform designs, handle budgets, coordinate the progress of projects with MBHS operating units and document and report project results to MBHS accountable leaders. Members of the Best Care Committee are: The vice president for health care improvement (as chair), the extant quality directors from each MBHS hospital and the employed physician group, the MBHS vice-president of risk management, representatives of the MBHS chief information officer and chief financial officer, physician quality leaders and nursing leadership council members and the physician and pharmacist leaders of the MBHS pharmacy and therapeutics committee. The MBHS chief executive officer, senior vice-president of clinical integration and senior vice-president of health care research and improvement/chief quality officer were invited to attend the committee meetings and received all committee materials (Ballard, 2003).

The ad hoc committee also recommended creating incentives to facilitate the expedient achievement of Best Care objectives. One such incentive used health care improvement performance management compensation tools in the extant MBHS performance compensation program, which was performed according to fiscal performance and patient satisfaction measures. The ad hoc committee insisted that such incentives be linked to specific clinical indicators, such as who had the lowest scores among medication mistake reporting. The need to evaluate the level of medication errors at MBHS proved an early opportunity to test these clinical indicators in examining drug underuse, overuse and misuse (errors), as well as Adverse Drug Events (ADE). In each case MBHS used the indicators to create baseline data, after which a specific quality improvement process was performed and the indicators were used to measure its efficiency.

As CEO, the responsibility for ensuring the ad hoc committee functioned appropriately and the clinical indicators were reported accurately could be delegated to the chief nursing officer or not. If it is delegated, however, it is important to keep in mind that in the end, it is the CEO's responsibility for the results of the data and keeping a close eye on the program may be preferable than delegation. The CEO must realize that real time reporting is essential in capturing medication errors and many errors slip by as nurses are afraid of punitive actions, dismissal and losing their licenses, so they do not report medication errors unless they have to. Many medication errors go unreported. It is the CEO's responsibility to change this and foster an atmosphere that will encourage reporting of all medication errors so that data will be correct and first and foremost patient safety will not be jeopardized.

\section{Conflict Management Strategies}

Leaders have a critical responsibility for managing conflicts when they arise, whether it is between staff or a patient issue, a supplier issue, or a reimbursement issue. Understanding the main methods of conflict management will help the manager become better prepared to deal with conflicts when they do arise. Conflict management strategies should begin with understanding the basics of conflict management. There are five basic conflict management strategies: Competition, avoidance, compromise, accommodation and collaboration. Competition involves using assertive, uncooperative approaches to a conflict to develop a winlose situation (Borkowski, 2009). For example, the supervisor may use negative performance evaluations and persuasion of his or her supervisor to manage the conflict to his or her desire. This type of conflict management leaves little balance and control for the employee. Collaboration involves highly assertive, cooperative behavior and develops a win-win situation. For example, if the issue is complex, the manager may seek input from the employees to find alternative solutions. Compromising uses both assertive and cooperative behaviors; both partners typically give up something to reach an agreement. For example, this type of conflict management would be appropriate when both parties cannot find a suitable agreement and the end result brings both or one better than or at least no worse off than they were before the conflict began. Accommodating uses unassertive and cooperative behaviors and is the opposite of competing. 
Table 1. Description of patients and medication orders

\begin{tabular}{llll}
\hline Items & AICU & CICU & Total \\
\hline Number of admissions & 304 & 326 & 630 \\
Number of unique patients & 294 & 322 & 610 \\
Patient age in years: mean \pm SD & $59 \pm 18$ & $64 \pm 13$ & $61 \pm 16$ \\
Patient gender: \% Female & 44 & 43 & 43 \\
Patient ethnicity: \% White & 94 & 96 & 95 \\
Days in the ICU: mean \pm SD; range & $9 \pm 9 ; 1-67$ & $5 \pm 4 ; 1-35$ & $7 \pm 7 ; 1-67$ \\
Study period in days & 119 & 72 & 191 \\
Patient-days & 2643 & 1504 & 4417 \\
Medication orders & 27,817 & 17,841 & 45,658 \\
Orders per patient-day: mean \pm SD & $11.4 \pm 4.4$ & $12.4 \pm 5.0$ & $11.9 \pm 4.8$ \\
Orders per admission: mean \pm SD & $91.5 \pm 88.0$ & $54.7 \pm 46.8$ & $72.5 \pm 72.1$ \\
\hline
\end{tabular}

This style is best used in short-term situations where temporary solutions are needed quickly to eliminate potentially volatile situations. Avoiding uses unassertive and uncooperative behaviors and is the opposite of collaborating (Borkowski, 2009). Managers that use this style typically want the conflict to work it out without interference.

\section{Positive Response for School Nurse Mission}

Most research on conflict management is focused on understanding the conditions under which the manager successfully resolves the conflict and comprehensively examining the dispute that may affect the outcomes of the negotiation. Strategic decisions are sometimes based on anticipation of future outcomes and consequences of the decisions made within the conflict management process itself. When a conflict occurs, the management method may have a significant impact on the outcome of the conflict (Melin, 2011). It is up to the manager to decide the appropriate method to handle the conflict, but the manager must be careful to apply the fundamentals of conflict management in an appropriate and professional manner.

The players in the Riverbend City Hospital School Nurse Mission were varied in their opinions on how to approach emergency credentialing. Unfortunately for Dr. Yee, Mrs. Garcia-Chavez and Mr. Lauderback were relying on the Good Samaritan Law to protect the hospital from liability despite the request made by the Emergency Coordinator, Jodi Lundquist and the Chief Executive Officer, Eugene Pittman, to send all volunteers to emergency credentialing. Although $\mathrm{Mr}$. Lauderback was not at the meeting, Mrs. Garcia-Chavez and Dr. Yee were and clearly heard both Lundquist and Pittman discuss the importance of emergency credentialing. During the conversation in the Emergency Room (ER), Dr. Yee made inappropriate remarks about Jodi Lundquist and the fact that she was new to the position and did not know her job duties. The three employees blatantly ignored the requirement to get emergency credentialing for Mary Jackson.

Conflict can arise from personality differences, differences in beliefs and stem from many sources in organizations including disrespecting a new employee, misjudging someone's credentials and group distrust. What Dr. Yee, Mrs. Garcia-Chavez and Mr. Lauderback felt was a combination of group distrust, disrespecting a new employee and misjudging someone's credentials. Conflict, however, is a powerful modifier for change (Bakhare, 2010). Mr. Pittman's role, along with Carol Yeager, the Chief Legal Consultant and Marcus Bertrand, the Chief Financial Officer, was to decide how to manage the conflict that arose as a result of the school nurse in in the ER. Using the conflict management tool of collaboration, they began to try to discover the facts of the conflict and resolve some of the issues surrounding the incident, which was the appropriate step and method. Managers can use collaboration to affect positive effects in conflicts by instituting change, avoiding group thinking, fostering creativity and innovation, creating a minimal level of conflict and raising and addressing problems. Mr. Pittman and the others were using a tactic called goal sharing where clear cut goals were outlined and identified. This technique is useful when managers have employees who violate rules and need reminders of goals and objectives and help with clarification (Bakhare, 2010). A combined use of collaboration and goal clarification would be successful in managing this conflict. A punishment would have to be delivered to the three staff members for violating the emergency plan. In addition to the punishment, the staff would need to be acknowledged as valued leaders and caregivers in the organization.

\section{Conclusion}

Public health of the US patient population is a primary target for QI programs targeting adverse drug events. It is the role of management to develop adequate programs to educate and protect the patient populations they are responsible for. A combination of conflict management strategies and negotiation efforts were used by the leaders at Riverbend City Hospital to manage the conflict caused by the nurse Mary Jackson who worked in the ER without the proper emergency credentialing. The leaders used the appropriate collaborative conflict management techniques to handle the conflict. The issue 
was discussed in detail and every perspective of the incident was gathered by the leaders. The leaders should be commended on their use of evidence-based, research proven techniques for conflict management. Other strategies for conflict management were not appropriate in this situation and would have led to confusion and possibly a worsening of the situation in the future. By using proven strategies for conflict management, the leaders of Riverbend City Hospital were able to solve this conflict professionally.

Medication errors are common and can lead to much suffering for the patient and nurse and expense to the health care system. Johnson and Young think that a medication error is a failure in the medical process that results in or has the potential to bring harm to a patient. Medication management reflects a high-risk activity in nursing; $78 \%$ of nurses have confessed that they ever made medication errors. An understanding of the situation and the process of medication errors help find areas for improvement within the activity.

Conflict occurs in all areas of daily life. Conflict can be described as when one person has a disagreement with another person or group over ideas or actions. Conflicts should be handled using the best technique for the type of conflict and with the outcome firmly in mind. Positive results can come from conflict, however the person who handles the conflict must understand the dynamics of conflict management and apply principles of intervention to prevent harm to the relationships of the individuals involved in the conflict and maintain a balance within the organization. For this to happen, many managers utilize several conflict management techniques depending on how the conflict is playing out, who is involved in the conflict and what the conflict is about. With certainty, conflict management can prevent irreparable harm to the organizational team spirit and the cohesion of the group so that work can get done and patient satisfaction and patient care do not suffer. Managers have a key responsibility in keeping conflicts out of the workplace and keeping employees focused on patient care. To do this, early intervention in any conflict is necessary and using evidence-based traditional methods of conflict management will help keep employees from becoming statistics. The purpose of this essay is to examine strategies to avoid conflict based on a scenario at Riverbend City Schools.

The following aspects will be authors' future or further research: (1) Predictive analytics based on big data to speed patient diagnosis, predict outcomes and efficiently manage patient population; (2) medication error reduction based on Radio Frequency Identification (RFID). RFID can help nurses monitor patients, detect medically significant events and link the right patient with the right medicine at the right time.

\section{Acknowledgment}

Thanks to all nurses and medical staff who took part in this study. This study was supported in part by Technology and Healthcare Solutions, Inc. in Mississippi, USA. No conflict of interest to disclose.

\section{Funding Information}

The authors have no support or funding to report.

\section{Author's Contributions}

All authors equally contributed in this study.

\section{Ethics}

This article is original and contains unpublished material. The corresponding author confirms that all of the other authors have read and approved the manuscript and no ethical issues involved.

\section{References}

Arinal, M.F., T. Cohn and C. Avila-Quintana, 2014. Evaluating the impact of medication cabinets in patients' rooms on a medical-surgical telemetry unit. MEDSURG Nurs., 23: 77-119. PMID: 24933783

Au, A., B. Duncan, H. Nicky, M. Barnes and D. Gurau et al., 2014. Errors are part of being human, but should we accept the cost of medication errors to patients/families? Dynamics Critical Care, 25: 43.

Bakhare, R., 2010. The manager and conflict management. SCMS J. Ind. Manag., 7: 41-57.

Ballard, D.J., 2003. Indicators to improve clinical quality across an integrated health care system. Int. J. Quality Health Care, 15: 13-23.

DOI: 10.1093/intqhe/mzg080

Borkowski, N., 2009. Organizational Behavior in Health Care. 2nd Edn., Jones and Bartlett Learning, ISBN-10: 0763763837, pp: 420.

Carayon, P., T.B. Wetterneck, R. Cartmill, M.A. Blosky and J. Walker et al., 2014. Characterising the complexity of medication safety using a human factors approach: an observational study in two intensive care units. BMJ Quality Safety, 23: 56-65.

DOI: $10.1136 /$ bmjqs-2013-001828

Cheung, K.C., W. Van Der Veen, M.L. Bouvy, M. Wensing and P.M. Van Den Bemt et al., 2014. Classification of medication incidents associated with information technology. 21: 63-70.

DOI: 10.1136/amiajnl-2013-001818 
Cannoy, S.D. and P.E. Carter, 2011. Information politics in health information exchange networks. 7: 65-90. DOI: $10.1080 / 15536548.2011 .10855911$

Flemons, W.W. and J.M. Davies, 2011. Patients are harmed (again), fingers point. What's wrong? Canadian Med. Associat. J., 183: 520-521. DOI: $10.1503 / \mathrm{cmaj} .101389$

Glasgow, J., 2011. Introduction to Lean and Six Sigma approaches to quality improvement. Agency Healthcare Research Quality: Advancing Excellence in Health Care.

Hamrick, H.E., S. Tutkus, A. Carbo, A. Tess and D.W. Bates et al., 2008. Medication safety messages for patients via the web portal: The med check intervention. Int. J. Med. Informat., 77: 161-168. DOI: 10.1016/j.ijmedinf.2007.04.007

Iselin, E.R., J. Sands and L. Mia, 2011. Multiperspective performance reporting systems, continuous improvement systems and organisational performance. J. Gen. Manag., 36: 19-36.

Johnson, M. and H. Young, 2011. The application of Aronson's Taxonomy to medication errors in nursing. J. Nurs. Care Quality, 26: 128-135. DOI: $10.1097 / \mathrm{NCQ} .0 \mathrm{~b} 013 \mathrm{e} 3181 \mathrm{f54b14}$

JC, 2012. National patient safety goals. Joint Commission.

Lövborg, H., M. Holmlund and S. Hägg, 2014. Medication errors related to transdermal opioid patches: Lessons from a regional incident reporting system. BMC Pharmacol. Toxicol., 15: 31.

DOI: $10.1186 / 2050-6511-15-31$
Mainz, J., 2003. Defining and classifying clinical indicators for quality improvement. Int. J. Quality Health Care, 15: 523-530. DOI: $10.1093 /$ intqhe/mzg081

McMillian, J.A., 2011. Quality improvement: name it: Analyzing systems and performance continually improves care. Contemporary Pediatrics, 28: 8-9.

Melin, M.M., 2011. The impact of state relationships on if, when and how conflict management occurs. Int. Stud. Quarterly, 55: 691-715. DOI: $10.1111 /$ j.1468-2478.2011.00665.x

Meredith, E.K., 2011. Developing frontline Staff in performance improvement: Part 1: tools for success. Nurs. Leader, 9: 50-53. DOI: $10.1016 /$ j.mnl.2011.01.015

NQMC, 2011. Medication reconciliation post-discharge: percentage of discharges from January 1 to December 1 of the measurement year for members 66 years of age and older for whom medications were reconciled on or within 30 days of discharge. National Quality Measures Clearinghouse.

Wright, K., 2014. The rules and regulations for medication management. Nurs. Resident. Care, 16: 232-235. DOI: 10.12968/nrec.2014.16.4.232

WHO, 2011. Latest developments in pharmacovigilance. WHO Drug Information, World Health Organization, 25: 33-36. 